\title{
Estimation of the Free Space in Front of a Moving Vehicle
}

\author{
Christian Lundquist, Thomas B. Schön \\ Linköping University, Linköping, Sweden
}

Copyright (C) 2009 SAE International

\begin{abstract}
There are more and more systems emerging making use of measurements from a forward looking radar and a forward looking camera. It is by now well known how to exploit this data in order to compute estimates of the road geometry, tracking leading vehicles, etc. However, there is valuable information present in the radar concerning stationary objects, that is typically not used. The present work shows how radar measurements of stationary objects can be used to obtain a reliable estimate of the free space in front of a moving vehicle. The approach has been evaluated on real data from highways and rural roads in Sweden.
\end{abstract}

\section{INTRODUCTION}

For a collision avoidance system it is imperative to have a reliable map of the environment surrounding the host vehicle. This map, consisting of both stationary and moving objects, has to be built in real time using measurements from the sensors present in the host vehicle. This is currently a very active research topic within the automotive industry and many other areas as well. Great progress has been made, but much remains to be done. Current stateof-the-art when it comes to the problem of building maps for autonomous vehicles can be found in the recent special issues [3-5] on the 2007 DARPA Urban Challenge. In these contributions measurements from expensive and highly accurate sensors are used, while we in the present paper utilize measurements from off-the-shelf automotive radars.

In this contribution we consider the problem of estimating the free space in front of the vehicle, making use of radar measurements originating from stationary objects. The free space is defined as the space where a ground vehicle can manoeuvre without colliding with other objects. Another name for the free space is the drivable space.

The present solution makes use of an already existing sensor fusion framework [19], which among other things provided a good road geometry estimate. This framework improves the raw vision estimate of the road geometry by fusing it with radar measurements of the leading vehicles. The idea is that the motion of the leading vehicles reveals information about the road geometry $[9,10,32]$. Hence, if the leading vehicles can be accurately tracked, their motion can be used to improve the road geometry estimates. Furthermore, we used a solid dynamic model of the host vehicle allowing us to further refine the estimates by incorporating several additional proprioceptive sensor measurements readily available on the CAN bus. The resulting, rather simple, yet useful map of the environment surrounding the host vehicle consists in

- Road geometry, typically parameterized using road curvature and curvature rate.

- Position and velocity of the leading vehicles.

- Host vehicle position, orientation and velocity.

This information can and has indeed been used to design simpler collision avoidance systems. However, in order

The Engineering Meetings Board has approved this paper for publication. It has successfully completed SAE's peer review process under the supervision of the session organizer. This process requires a minimum of three (3) reviews by industry experts.

All rights reserved. No part of this publication may be reproduced, stored in a retrieval system, or transmitted, in any form or by any means, electronic, mechanical, photocopying, recording, or otherwise, without the prior written permission of SAE.

ISSN 0148-7191

Positions and opinions advanced in this paper are those of the author(s) and not necessarily those of SAE. The author is solely responsible for the content of the paper.

SAE Customer Service:

SAE Web Address:

Printed in USA
Tel: $\quad$ 877-606-7323 (inside USA and Canada)

Tel: $\quad$ 724-776-4970 (outside USA)

Fax: $\quad 724-776-0790$

Email: CustomerService $₫$ sae.org 
to devise more advanced systems, more information about the environment surrounding the host vehicle is needed. The purpose of this paper is to exploit information already delivered by the radar sensor in order to compute a more complete map. Hence, there is no need to introduce any new sensors, it is just a matter of making better use of the sensor information that is already present in a modern premium car. To be more precise, it is the radar echoes from stationary objects that are used to estimate the road borders, which determines the free space in front of the host vehicle. The radar measurements used originate from for instance, guard rails and concrete walls. Obviously these stationary radar measurements are not enough to fully explain the road borders. However, as we will see, there is surprisingly much information present in these measurements.

The key in our approach is to make use of the road curvature estimate from the sensor fusion framework [19] mentioned above to sort the stationary radar measurements according to which side of the road they originate from. These measurements are then used together with the estimates from the sensor fusion to dynamically form a suitable constrained quadratic program (QP) for estimating the free space in front of the vehicle. This QP models the temporal correlation that exists in roads and the fact that the road shape cannot change arbitrarily fast.

The approach has been evaluated on real data from highways and rural roads in Sweden. The test vehicle is a Volvo S80 equipped with a forward looking $77 \mathrm{GHz}$ mechanically scanned FMCW radar and a forward looking vision sensor (camera).

\section{RELATED WORK}

We have also investigated a completely different approach to represent the map of the free space in front of the host vehicle based on so call occupancy grid maps (OGM). This is a commonly used method for tackling the problem of generating consistent maps from uncertain measurements of stationary object under the assumption that the host vehicle pose is known. Occupance grid maps are very popular in the robot community, especially for all sorts of autonomous vehicles equipped with laser scanners, indeed several of the DARPA vehicles [3-5] used OGM's. The OGM was introduced by Elfes [7] and a solid treatment can be found in the recent textbook [28].

The map is discretized into a number of cells with an associated probability of occupancy. The map is represented by a matrix, with each element corresponding to a map-cell. Figure 1a shows an OGM computed for the highway situation given in the host vehicle's camera view in Figure 1b. The host vehicle is positioned at $(200,200)$,

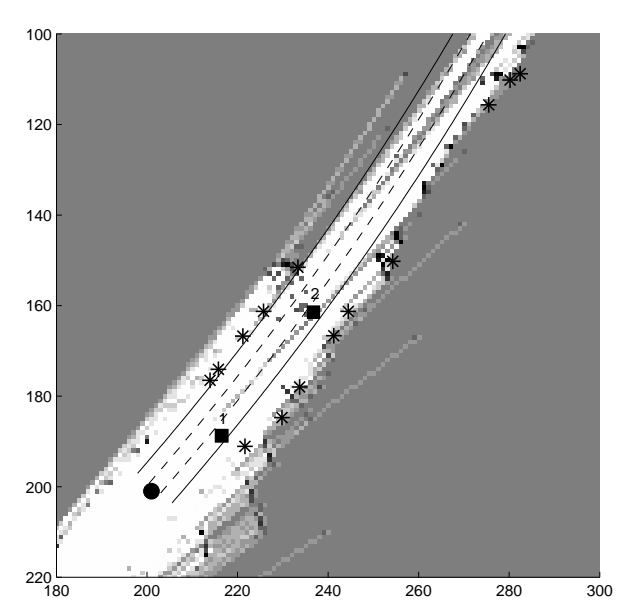

(a)

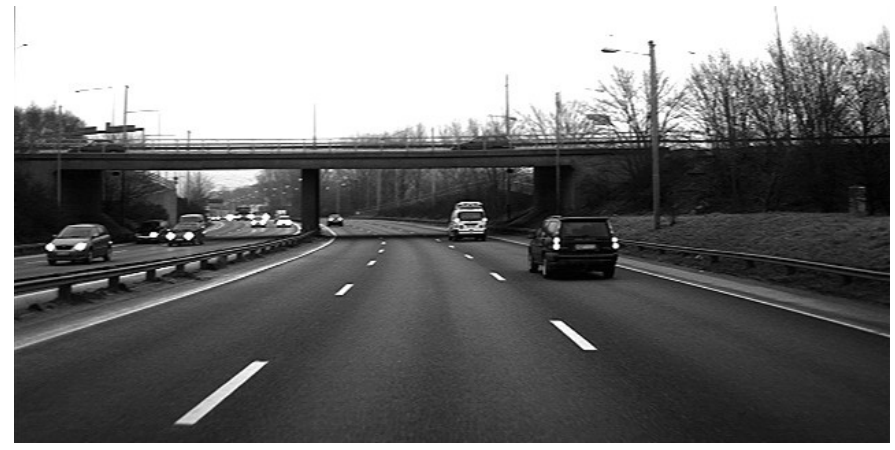

(b)

Figure 1: The filled circle at position $(200,200)$ in the occupancy grid map in Figure (a) is the host vehicle, the stars are the radar observations obtained at this time sample, the black squares with numbers 1 and 2 are the two leading vehicles that are currently tracked. The gray-level in the figure indicates the probability of occupancy, the darker the grid cell, the more likely it is to be occupied. The shape of the road is given as solid and dashed lines, calculated as described in [19]. The camera view from the host vehicle is shown in Figure (b), the concrete walls, the guardrail and the pillar of the bridge are interesting landmarks. Furthermore, the two tracked leading vehicles are clearly visible in the right lane.

indicated by the filled circle. The gray-level in the occupancy map indicates the probability of occupancy, the darker the grid cell, the more likely it is to be occupied. As can be seen in Figure 1a, the OGM generates a goodlooking overview of the traffic situation. However, since the measurements are obtained from a standard automotive radar the results are not very informative for a collision avoidance system, better accuracy is needed. For a more complete description of the application of the OGM to the present problem we refer to [18].

The work presented here is clearly related to lane tra- 


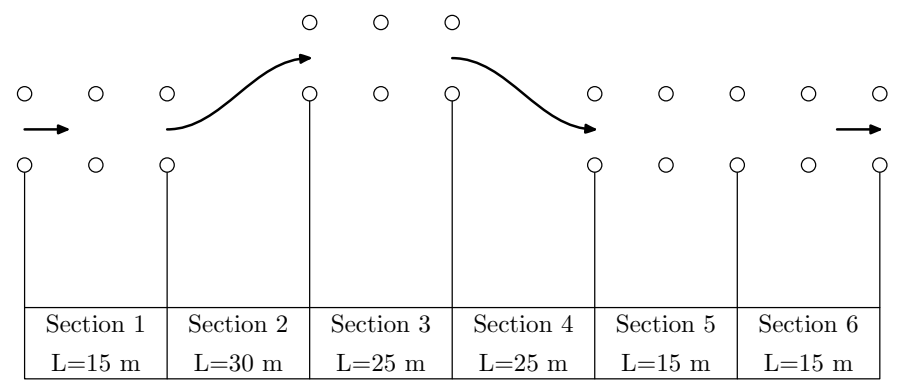

Figure 2: ISO 3888 double lane change maneuver [13].

cking, which by now is a very well-studied problem, see e.g. [23] for a recent survey using cameras. In fact the required sensor fusion framework [19] makes use of the estimates from a visual lane tracker. The recent book [6] contains a lot of interesting information about detecting and tracking lanes using cameras. Lane tracking has also been tackled using radar sensors, see e.g., [14, 17, 21, 24] and laser sensors, see e.g. [31]. Using laser scanners there have been several approaches making use of reflections from the road boundary, such as crash barriers and reflection posts, to compute information about the free space, see e.g. $[15,16,26]$. Furthermore, the use of a side looking radar to measure the lateral distance to a sidewall is described in various papers, e.g., [8,22,27]. The intended application in these papers $[8,22,27]$ were automatic lateral control. Here, we have no specific application in mind, we just try to obtain the best possible map based on the available sensor information. This map can then be used by any control system.

In [30] the authors presents an algorithm for free space estimation, capable of handling non-planar roads, using a stereo camera system. Similar to the present paper the authors make use of a parametric model of the road ahead. An interesting avenue for future work is to combine the idea presented in this paper with the ideas of [30] within a sensor fusion framework.

\section{PROBLEM FORMULATION}

An important question is how the information of the free space should be represented and for which distances ahead of the vehicle that it is needed. We will start by addressing the latter through an example, the standard double lane change manoeuvre according to ISO 3888 [13]. In this maneuver a vehicle has to overtake an obstacle and come back to its original lane as shown in Figure 2. Assume that the host vehicle is entering section 1 at a velocity of $100 \mathrm{~km} / \mathrm{h}$ and that there is an obstacle straight ahead in section 3. The free space, i.e. the distance to the left and right road borders has to be known in order to autonomously overtake the obstacle as shown in the fi- gure. This means that an automatic collision avoidance system needs to have information about the free space at least three sections ahead in order to make a decision on where to steer the vehicle. From this simple, yet informative, calculation we conclude that the road must be well estimated for at least $60 \mathrm{~m}$ ahead when driving at approximately $100 \mathrm{~km} / \mathrm{h}$.

In this paper we will use the planar coordinate transformation matrix

$$
A^{R L}=\left(\begin{array}{cc}
\cos \psi & -\sin \psi \\
\sin \psi & \cos \psi
\end{array}\right)
$$

to transform a vector, represented in the vehicle's coordinate system $L$, into a vector, represented in the reference coordinate system $R$, where $\psi_{L R}$ is the angle of rotation from $R$ to $L$. We will refer to this angle as the yaw angle of the vehicle, and in order to simplify the notation we will use $\psi \triangleq \psi_{L R}$. The point $O$ is the origin of $R$ and $P$ is the origin of $L$ situated in the vehicles center of gravity. The geometric displacement vector $r_{P O}^{R}$ is the direct straight line from $O$ to $P$ represented with respect to the frame $R$. The angles and distances are shown in Figure 3.

A stationary object $i$ will be referred to as an observation in the point $S_{i}$. The radar in the host vehicle measures the azimuth angle $\psi_{S_{i} P}$ and the range $r=\left\|r_{S_{i} P}^{L}\right\|_{2}$ to the

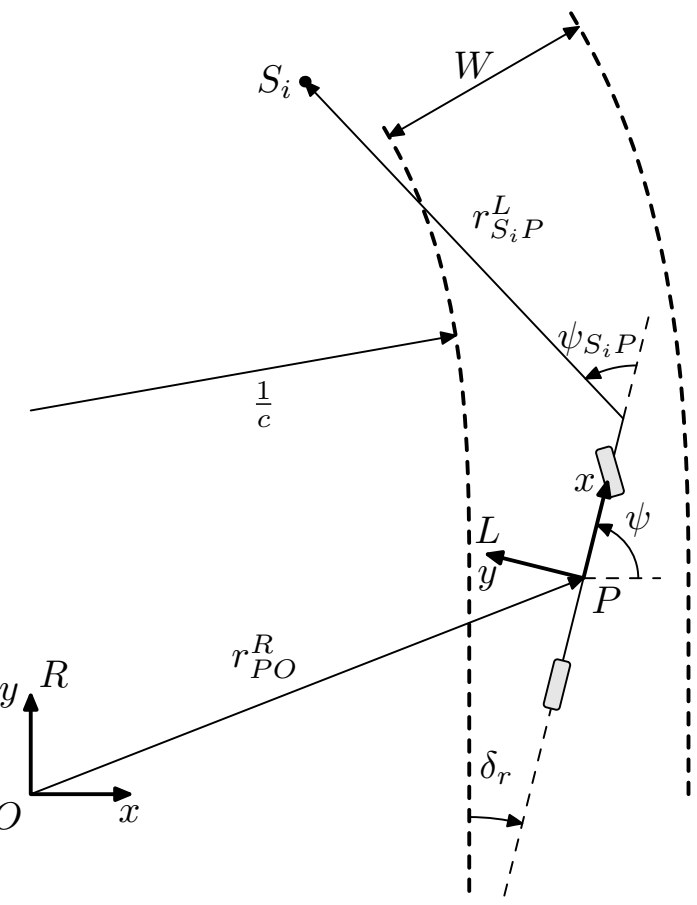

Figure 3: The host vehicle's coordinate frame $L$ has its origin $P$ situated in the vehicle's center of gravity. A stationary object $S_{i}$ is observed at a distance $\left\|r_{S_{i} P}^{L}\right\|_{2}$ and an angle $\psi_{S_{i} P}$ with respect to the vehicles radar, which is mounted in the radiator cowling. The lane width is $W$, the heading angle is $\delta_{r}$ and the road curvature is $c$. 
stationary object. These are transformed into Cartesian coordinates $\left(x_{S_{i} O} \quad y_{S_{i} O}\right)^{T}$ in any coordinate frame.

All the observations of stationary objects $S=\left\{S_{i}\right\}_{i=1}^{N_{s}}$ from the radar are sorted into two ordered sets, one for the left side $S_{1}$ and one for the right side $S_{\mathrm{r}}$ of the road. In order to be able to perform this sorting we need some information about the road geometry, otherwise it is of course impossible. In [19] we provide a sensor fusion framework for sequentially estimating the parameters $l, \delta_{r}, c_{0}$ in the following model of the road's white lane markings,

$$
y^{L}=l+\delta_{r} x^{L}+\frac{c_{0}}{2}\left(x^{L}\right)^{2},
$$

where $x^{L}$ and $y^{L}$ are expressed in the host vehicle's coordinate frame $L$. The angle between the longitudinal axis of the vehicle and the road lane is $\delta_{r}$, see Figure 3. It is assumed that this angle is small and hence the approximation $\sin \delta_{r} \approx \delta_{r}$ is used. The curvature parameter is denoted by $c_{0}$ and the offset between the host vehicle and the white lane is denoted by $l$.

The information about the road shape in (2) can now be used to decide if an observation should be sorted into the left set according to

$$
S_{1}=\left\{S_{i} \in S \mid y_{S_{i} P}^{L} \geq l+\delta_{r} x_{S_{i} P}^{L}+\frac{c_{0}}{2}\left(x_{S_{i} P}^{L}\right)^{2}\right\}
$$

or the right set according to

$$
S_{\mathrm{r}}=\left\{S_{i} \in S \mid y_{S_{i} P}^{L}<l+\delta_{r} x_{S_{i} P}^{L}+\frac{c_{0}}{2}\left(x_{S_{i} P}^{L}\right)^{2}\right\} .
$$

Observations which lay more than $200 \mathrm{~m}$ behind the vehicle are removed from the set. The two sets $S_{1}$ and $S_{\mathrm{r}}$ are resorted at every sample, according to the new curvature estimate.

Given the data in $S_{1}$ we seek a road border model, provided by a predictor

$$
\hat{y}_{S_{i} P}^{L}\left(x_{S_{i} P}^{L}, \theta\right),
$$

where $\theta$ denotes a parameter vector describing the road boarders. The exact form of this predictor is introduced in the subsequent section, where two different predictors are derived. The data in $S_{\mathrm{r}}$ in treated analogously. The road boarder parameters $\theta$ are estimated by solving the following least-square problem

$$
\min _{\theta} \sum_{i=1}^{N} \lambda_{i}\left(y_{S_{i} P}^{L}-\hat{y}_{S_{i} P}^{L}\left(x_{S_{i} P}^{L}, \theta\right)\right)^{2}
$$

where $N$ is the number of observations and $\lambda_{i}$ is a weighting factor. The problem (6) is formulated as if there is only an error in the $y$-coordinate. Obviously there are errors present also in the $x$-coordinate. This can be taken care of by formulating a so called errors-in-variables problem (within the optimization literature this problem is referred to as a total least squares problem), see e.g., [1]. However, for the sake of simplicity we have chosen to stick to an ordinary least squares formulation in this work.

\section{ROAD BORDER MODEL}

In this section we will derive and analyze two different predictor models, one linear and one nonlinear.

An important problem to be solved is to decide which radar measurements that should be used in estimating the parameters. Later in this section we will introduce suitable constraints that must be satisfied. This will allow us to remove non-relevant data, i.e., outliers.

PREDICTOR - The two ordered sets $S_{1}$ and $S_{\mathrm{r}}$ are handled analogously. Hence, only the processing related to the left set is described here. The observations are expressed in the reference coordinate system $R$ when they are stored in $S_{1}$. Obviously it is straightforward to transform them into the vehicle's coordinate system, using the rotation matrix $A^{L R}=\left(A^{R L}\right)^{T}$.

As depicted earlier the lanes are modeled using the polynomial (2). Let us assume that the white lane markings are approximately parallel with the road border. In order to allow the number of lanes to change, without simultaneously changing the curvature, we extend the second order model (2) with a fourth element. Hence, a linear predictor is provided by

$$
\hat{y}_{1}^{L}\left(x^{L}, \theta_{1}\right)=l_{0}+l_{1} x^{L}+l_{2}\left(x^{L}\right)^{2}+l_{3}\left(x^{L}\right)^{3},
$$

which is a third order polynomial, describing the road's left border, given in the host vehicle coordinate system.

By analyzing road construction standards, such as [29], we assume that the increment and decrement of the number of lanes can be modelled using the arctan function illustrated in Figure 4a. This allows for a continuous, but possible rapid, change in shape. Let us now, as a second approach, extend (2) and form the following nonlinear predictor

$$
\begin{aligned}
\hat{y}_{2}^{L}\left(x^{L}, \theta_{2}\right) & =l_{0}+l_{1} x^{L}+l_{2}\left(x^{L}\right)^{2} \\
& +k \arctan \tau\left(x^{L}-b\right),
\end{aligned}
$$

where the parameter $b$ indicates where arctan crosses zero. The slope $\tau$ and magnitude $k$ could be chosen according to typical road construction constants. An example of the complete nonlinear road border model (8) is shown in Figure $4 b$.

We will start describing the linear model (7) and come back to the nonlinear model (8) later in this section. Given 


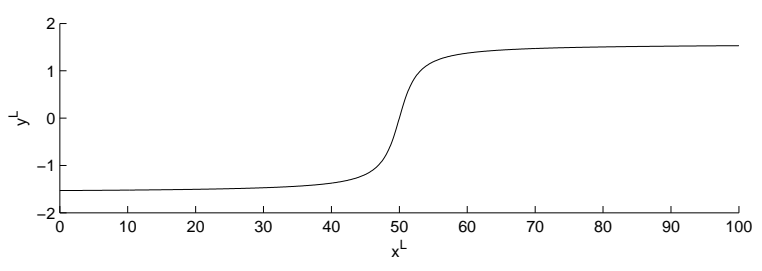

(a)

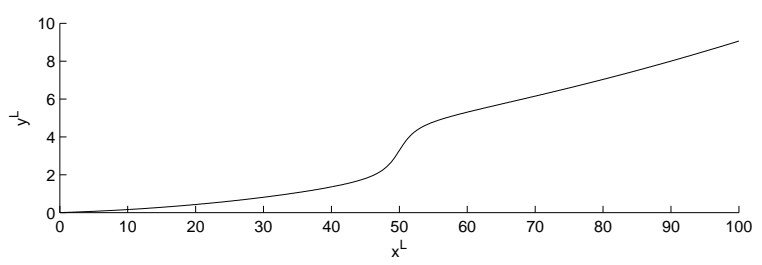

(b)

Figure 4: A pure arctan is shown in Figure (a), whereas the complete expression (8) is shown in Figure (b) for a typical example.

the $N_{l}$ observations in $S_{1}$, the parameters

$$
\theta_{l}=\left(\begin{array}{llll}
l_{0} & l_{1} & l_{2} & l_{3}
\end{array}\right)^{T}
$$

can be approximated by rewriting the linear predictor (7) according to

$$
\widehat{Y}_{1}^{L}=\left(\Phi^{L}\right)^{T} \theta_{l},
$$

where the regressors $\left(i=1, \ldots, N_{l}\right)$

$$
\varphi_{i}^{L}=\left(\begin{array}{llll}
1 & x_{S_{i} P}^{L} & \left(x_{S_{i} P}^{L}\right)^{2} & \left(x_{S_{i} P}^{L}\right)^{3}
\end{array}\right)^{T} .
$$

are stacked on top of each other in order to form

$$
\Phi^{L}=\left(\varphi_{1}^{L}, \ldots, \varphi_{N_{l}}^{L}\right),
$$

The parameters are found by minimizing the weighted least square error (6), here in matrix form

$$
\left\|Y^{L}-\Phi^{L} \theta_{l}\right\|_{\Lambda}^{2}=\left(Y^{L}-\Phi^{L} \theta_{l}\right)^{T} \Lambda\left(Y^{L}-\Phi^{L} \theta_{l}\right),
$$

where $\Lambda$ is a weighting matrix

$$
\Lambda=\operatorname{diag}\left(\begin{array}{lll}
\lambda_{1} & \cdots & \lambda_{N_{l}}
\end{array}\right) .
$$

and the $y$-coordinates are given by

$$
Y^{L}=\left(y_{1}^{L}, \ldots, y_{N_{l}}^{L}\right)^{T} .
$$

The right hand side of the road is modeled analogously, using the following parameter vector,

$$
\theta_{r}=\left(\begin{array}{llll}
r_{0} & r_{1} & r_{2} & r_{3}
\end{array}\right)^{T}
$$

The azimuth angle $\psi_{S_{i} O}$ is measured with lower accuracy than the range $r$ in the radar system. This influences the uncertainty of the measurements, when transformed into
Cartesian coordinates in accordance to the measured distance. Therefore, the elements of the weight matrix $\Lambda$ in (13) are defined as

$$
\lambda_{i}=\frac{1}{\log r_{i}}, \quad i=1, \ldots, N_{l},
$$

modelling the fact that stationary objects close to the vehicle are measured with higher accuracy than distant objects. Hence, the closer the object is, the hight the weight.

The problem of minimizing (13) can be rewritten as a quadratic program [2] according to

$$
\min _{\theta_{l}} \theta_{l}^{T} \Phi^{T} \Lambda \Phi \theta_{l}-2\left(Y^{L}\right)^{T} \Lambda \Phi \theta_{l}
$$

A straightforward solution of this problem will not work due to the simple fact that not all of the stationary objects detected by the radar stems from relevant objects for our purposes. For example, under some circumstances the radar also detects objects at the opposite side of the highway. These observations could for example stem from a guard rail or the concrete wall of a gateway from e.g. a bridge, see Figure $5 b$. If the road borders are estimated according to the quadratic program in (18) using these observations the result will inevitably be wrong. In order to illustrate that this is indeed the case the result is provided in Figure 5a. In the subsequent section we will explain how this situation can be avoided by deriving a set of feasibility conditions that the curve parameters $\theta_{l}$ and $\theta_{r}$ have to fulfill.

Let us briefly revisit the nonlinear model (8). Since this predictor is nonlinear, it cannot be factored in the same way as we did for the linear predictor in (10). Instead, we have to keep the nonlinear form, resulting in the following optimization problem to be solved

$$
\min _{\theta}\left\|Y^{L}-\widehat{Y}_{2}^{L}\left(X^{L}, \theta_{2}\right)\right\|_{\Lambda}^{2},
$$

where $Y^{L}$ was defined in (15) and similarly $\widehat{Y}_{2}^{L}$ are the nonlinear predictions

$$
\begin{aligned}
\hat{y}_{2}^{L}\left(x^{L}, \theta_{2}\right) & =l_{0}+l_{1} x^{L}+l_{2}\left(x^{L}\right)^{2} \\
& +k \arctan \tau\left(x^{L}-b\right)
\end{aligned}
$$

stacked on top of each other. Hence, the parameters $\theta_{2}$ used in (19) are given by

$$
\theta_{2}=\left(\begin{array}{llllll}
l_{0} & l_{1} & l_{2} & k & \tau & b
\end{array}\right)^{T} .
$$

The resulting problem (19) is a non-convex least-squares problem. 


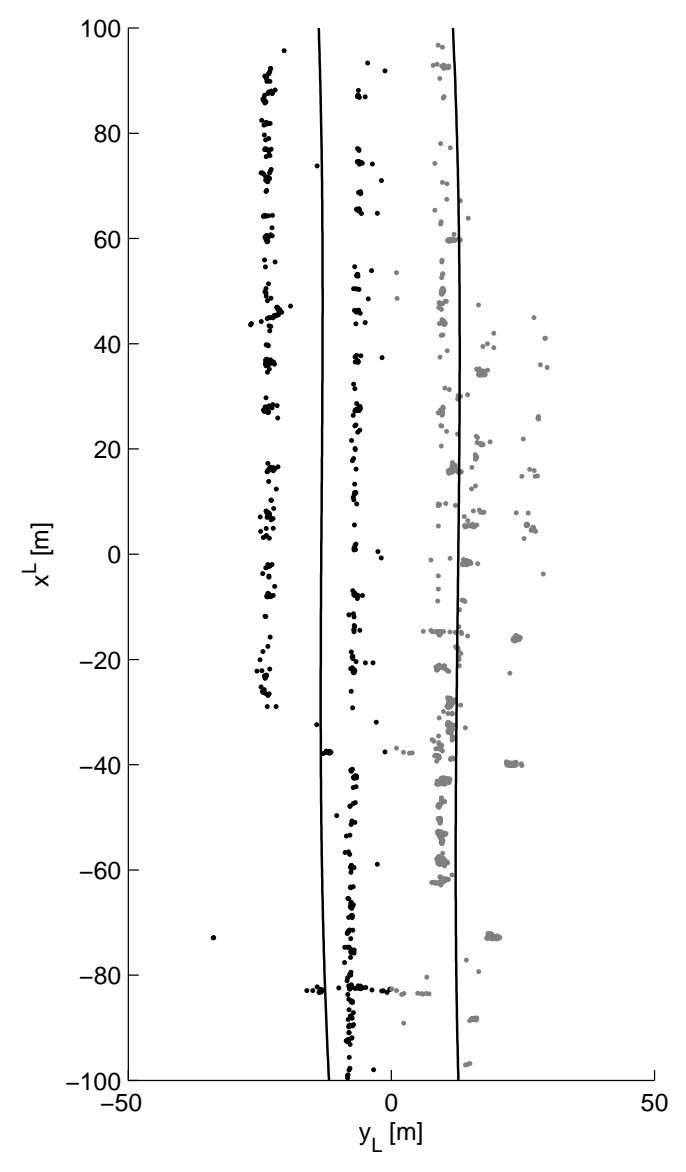

(a)

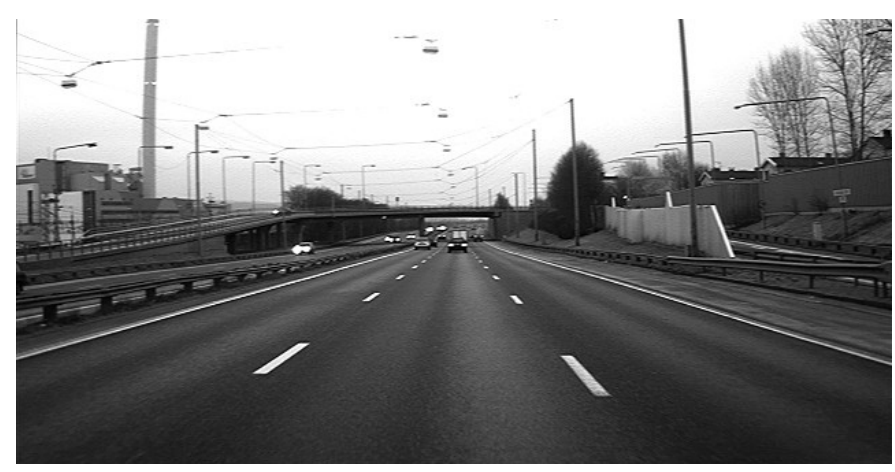

(b)

Figure 5: The gateway shown on the opposite side of the highway in Figure (b) misleads the road boarder estimation. The stored observations are shown together with the estimated road boarders (lines) in Figure (a). The black points belongs to the left set $S_{1}$ and the gray points belongs to the right set $S_{\mathrm{r}}$.

CONSTRAINING THE PREDICTOR - The predictor has to be constrained for the problem formulation to be interesting. More specifically, we will in this section derive constraints forming a convex set, guaranteeing that the resulting linear optimization problem remains quadratic. This problem can then be efficiently solved using a dual active set method* [11].

As we assume that the white lane markings (2) are approximately parallel with the road border (7), we could use the angle $\delta_{r}$ to constrain the second border parameter $l_{1}$ and we could use the curvature $c_{0}$ to constrain the third border parameter $l_{2}$ according to

$$
\begin{array}{cc}
(1-\Delta) \delta_{r}-\epsilon_{\delta_{r}} \leq l_{1} \leq(1+\Delta) \delta_{r}+\epsilon_{\delta_{r}} & \text { if } \delta_{r} \geq 0 \\
(1+\Delta) \delta_{r}-\epsilon_{\delta_{r}} \leq l_{1} \leq(1-\Delta) \delta_{r}+\epsilon_{\delta_{r}} & \text { if } \delta_{r}<0 \\
\frac{(1-\Delta) c_{0}-\epsilon_{c_{0}}}{2} \leq l_{2} \leq \frac{(1+\Delta) c_{0}+\epsilon_{c_{0}}}{2} & \text { if } c_{0} \geq 0 \\
\frac{(1+\Delta) c_{0}-\epsilon_{c_{0}}}{2} \leq l_{2} \leq \frac{(1-\Delta) c_{0}+\epsilon_{c_{0}}}{2} & \text { if } c_{0}<0
\end{array}
$$

where the allowed deviation $\Delta$ is chosen as $10 \%$, i.e., $\Delta=$ 0.1. A small value $\epsilon$ is added to avoid that both the upper and lower bounds are equal to 0 in case $\delta_{r}$ or $c_{0}$ is equal to 0 . Several different approaches for estimating the road curvature $c_{0}$ are described in [20].

The first border parameter $l_{0}$ is not constrained, because the number of lanes may change at e.g. a gateway. It should be possible for the border of the road to move in parallel to the host vehicles motion without any conditions.

In order to create a feasibility condition for the fourth parameter $l_{3}$ of the linear model, the estimated position of the host vehicle expressed in the reference frame $R$ is saved at each time sample. A data entry is removed from the set if it lays more than $200 \mathrm{~m}$ behind the current position. Furthermore, the estimated curvature is used to extrapolate points $200 \mathrm{~m}$ ahead of the vehicle. These points together with information about the host vehicle's earlier positions are used to derive a driven path as a third order polynomial

$$
y^{L}=l+\delta_{r} x^{L}+\frac{c_{0}}{2}\left(x^{L}\right)^{2}+\frac{c_{1}}{6}\left(x^{L}\right)^{3} .
$$

Especially the parameter $c_{1}$ is of interest and can be used to constrain $l_{3}$. Hence, the final inequality, which will further constrain (18) is given by

$$
\begin{array}{ll}
\frac{(1-\Delta) c_{1}-\epsilon_{c_{1}}}{6} \leq l_{3} \leq \frac{(1+\Delta) c_{1}+\epsilon_{c_{1}}}{6} & \text { if } c_{1} \geq 0, \\
\frac{(1+\Delta) c_{1}-\epsilon_{c_{1}}}{6} \leq l_{3} \leq \frac{(1-\Delta) c_{1}+\epsilon_{c_{1}}}{6} & \text { if } c_{1}<0 .
\end{array}
$$

To summarize, the constrained optimization problem to be

\footnotetext{
* The QP code was provided by Dr. Adrian Wills at the University of Newcastle, Australia, see http: / / sigpromu . org/quadprog. This code implements the method described in $[12,25]$.
} 
solved based on the linear predictor (7) is given by

$$
\begin{array}{cc}
\min _{\theta_{1}} & \left\|Y^{L}-\widehat{Y}_{1}^{L}\left(X^{L}, \theta_{1}\right)\right\|_{\Lambda}^{2} \\
\text { s.t. } & (22)
\end{array}
$$

The parameter $b$, of the nonlinear model (8) is constrained by the measurement distance and the parameters $k$ and $\tau$ are constrained by road construction standards. The resulting nonlinear least-squares problem is finally given by

$$
\begin{aligned}
& \min _{\theta_{2}}\left\|Y^{L}-\widehat{Y}_{2}^{L}\left(X^{L}, \theta_{2}\right)\right\|_{\Lambda}^{2} \\
& \text { s.t. } \\
& \text { (22) } \\
& b_{\max } \leq b \leq-b_{\max } \\
& k_{\max } \leq k \leq-k_{\max } \\
& \tau_{\max } \leq \tau \leq \tau_{\min } .
\end{aligned}
$$

OUTLIER REJECTION - The difference between the observed point and the calculated road border lines is used to separate and remove outliers which lie more than 1.5 lane width $(W)$ from the lines. Subsequently the quadratic program (18) is used a second time and the result is shown in Figure 6. For this case, the two predictor models yields approximately the same result.

An advantage of the nonlinear model is its ability to model changes in the number of lanes, as can be seen in Figure $7 \mathrm{a}$, where the number of lanes changes from two to three. Recall that it is the use of the arctan function that allows us to model changes in the number of lanes. The new lane originates from an access road to the highway. The corresponding camera view is shown in Figure $7 \mathrm{~b}$.

COMPUTATIONAL TIME - We have compared the computation time for the two proposed predictors with constraints. The nonlinear least square problem (26) was solved using the function fmincon in MATLABS optimization toolbox. Furthermore, we have used two different methods for solving the quadratic problem (25). The first method is the active set method mentioned earlier, where parts are written in C-code, and the second method used is quadprog in MATLABS optimization toolbox. The computational time was averaged over a sequence of 1796 samples. The sample time is $0.1 \mathrm{~s}$, implying that the measurements were collected during $179.6 \mathrm{~s}$ highway driving. The results are shown in Table 1.

The computation time of the nonlinear predictor is about $38 \%$ higher than it is for the linear predictor proposed in this paper. The MATLAB function quadprog needs $149 \%$ more computational time. This indicates that the computational time of the nonlinerar predictor can possibly be reduced by utilizing an optimized C-code implementation.

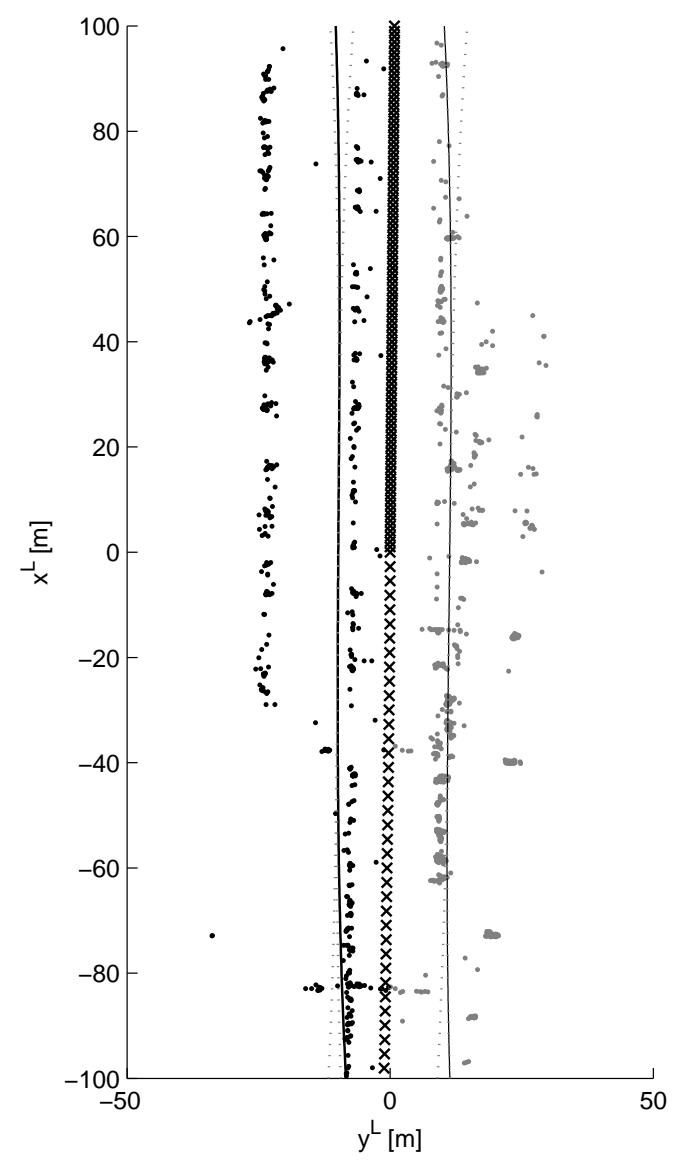

Figure 6: Road border estimation for the same situation as in Figure 5a, but the additional constraints are now used. The feasible set for the parameters $l_{1}, l_{2}$ and $l_{3}$ is between the dashed lines. The crosses shows the driven path (for $x<0$ ) and the estimated path (for $x>0$ ).

Table 1 Average computational time for one sample.

\begin{tabular}{|l|r|}
\hline Method & Time [ms] \\
\hline Linear Predictor (this paper) & 84 \\
Linear Predictor (quadprog) & 209 \\
Nonlinear Predictor & 116 \\
\hline
\end{tabular}

\section{CALCULATING THE FREE SPACE}

The free distance to the left and the right road borders is now easily calculated by considering the first parameters $l_{0}$ and $r_{0}$ respectively. The number of lanes on the left hand side is given by

$$
\max \left(\left\lfloor\frac{l_{0}-L}{W}\right\rfloor, 0\right)
$$




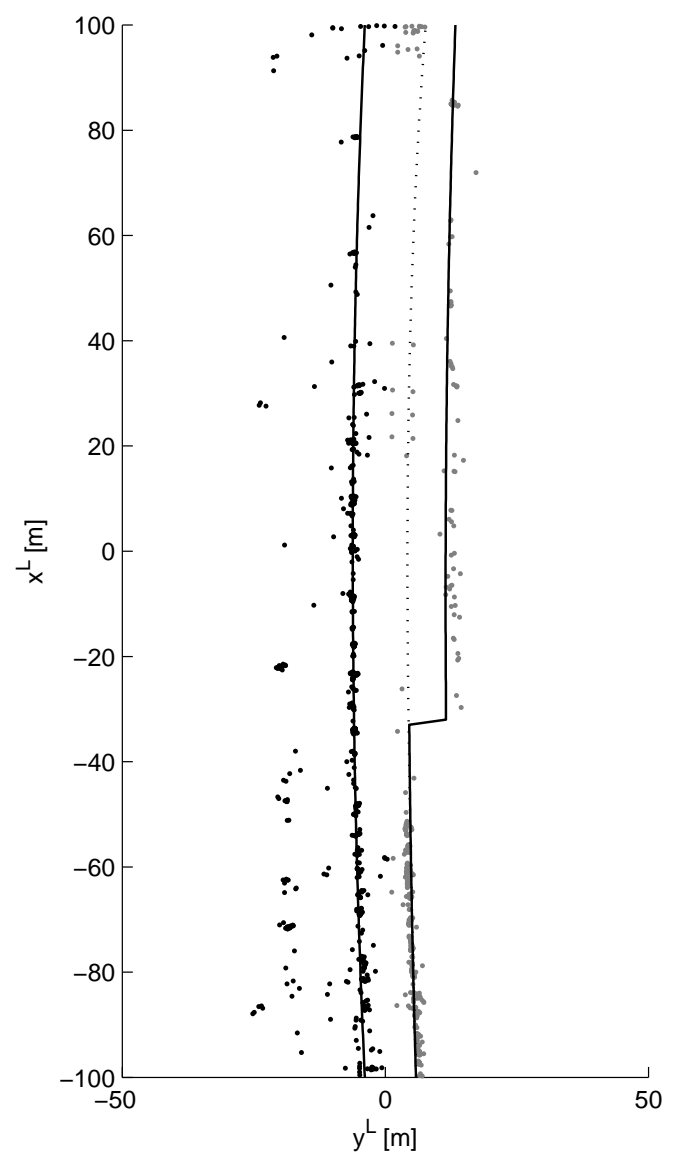

(a)

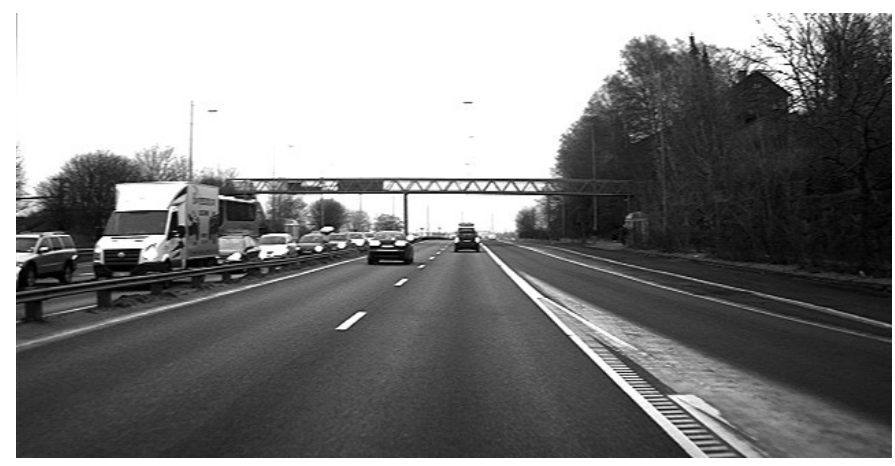

(b)

Figure 7: A change in the number of lanes is modeled accurately using the arctan function in the nonlinear predictor, as shown by the solid line in Figure (a). The dashed line is the result of the linear predictor. The camera view of the traffic situation is shown is Figure (b).

and the number of lanes on the right hand side is given by

$$
\max \left(\left\lfloor\frac{-r_{0}-R-2}{W}\right\rfloor, 0\right) .
$$

In the expressions above $L$ and $R$ are the distances from the sensor in the host vehicle to the left and right lane markings of the currently driven lane. We assume that the emergency lane is $2 \mathrm{~m}$ on the right hand side of the road [29].

The number of observed stationary objects depends on the surrounding environment. A guard rail or a concrete wall results in more observations than for example a forest. Hence, the estimated border lines are accompanied by a quality measure which depends on the number of observations and their variance. The variance is calculated before and after the outliers have been removed.

It is still a problem to detect the distance to the road border if there is a noise protection wall some meters to the right of the road. This wall generates many observations with small variance and cannot be distinguished from a guard rail. However, one solution might be to include camera information in a sensor fusion framework.

BORDER LINE VALIDITY - A very thrilling problem with the present curve fitting approach is that there are no gaps to properly leave or enter the road at a gateway. A collision avoidance system would brake the vehicle automatically if leaving the road at a gateway when simultaneously crossing the border line. This leads us to the conclusion that the border lines should only be defined if the number of observations around it lies above a certain limit.

In a first step we calculate the distance between the line and the observations in the set $S_{1}$

$$
d_{l, i}=\left|y_{i}^{L}-\left(\delta_{r} x_{i}^{L}+\frac{c_{0}}{2}\left(x_{i}^{L}\right)^{2}\right)\right| \quad \text { for } \quad i=1, \ldots, N_{l}
$$

and compare it with a constant or variable, e.g. the lane width $W$

$$
n_{i}= \begin{cases}1 & \text { if } d_{l, i}>W \\ 0 & \text { otherwise }\end{cases}
$$

In a second step the border line is segmented in valid and not valid parts. The start and end points of the valid parts are given by identifying the indices of two non equal and adjoined elements in the vector $n$. By applying the XOR function $(\oplus)$ according to

$$
c=n_{2: N_{l}} \oplus n_{1: N_{l}-1},
$$

the start and end points of the border line are identified as the indices with $c=1$. These indices are stored in two additional sets for the left and right border lines, respectively. An example is shown in Figure $8 \mathrm{a}$ and the corresponding camera view in Figure 8b. The gateway to the right leads to a gap in the right border line, between $48-73 \mathrm{~m}$ ahead of the host vehicle. One of the leading vehicles lies between the host vehicle and the guard rail, this is the reason whey there are so few stationary object on the left hand side from about $70 \mathrm{~m}$ ahead and why no line could be drawn. 


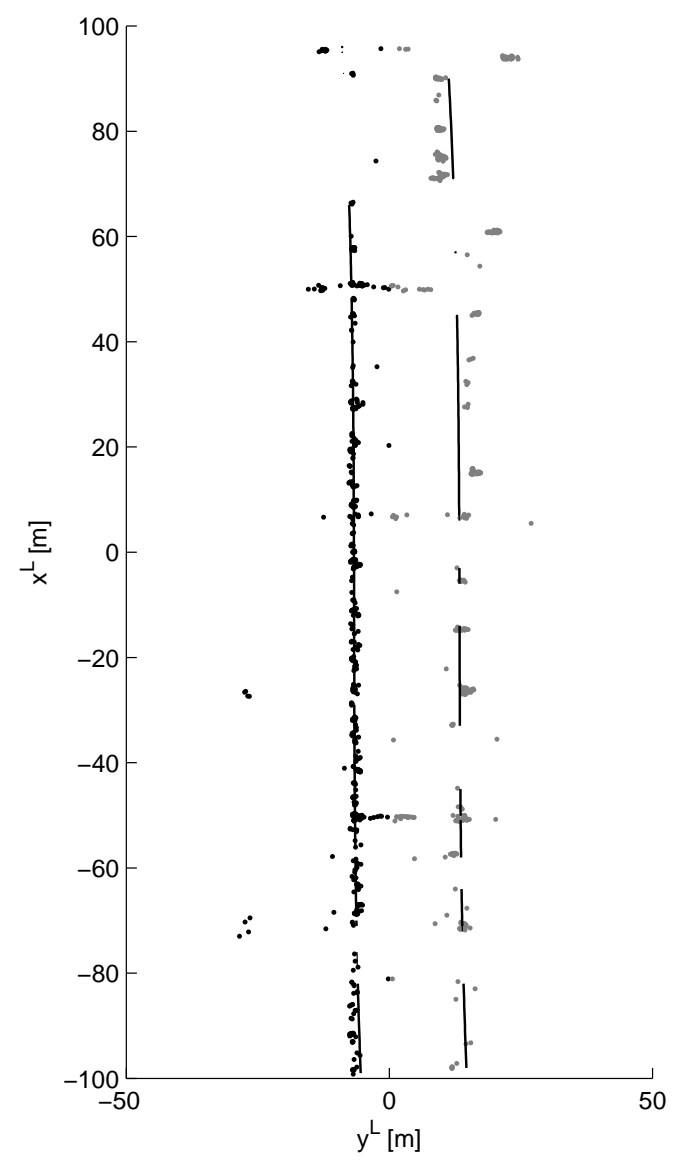

(a)

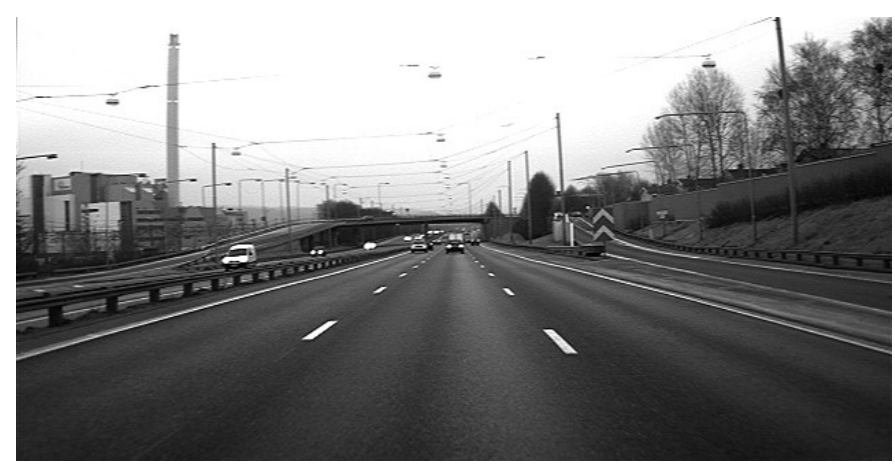

(b)

Figure 8: The gateway to the right in Figure (b) leads to a gap in the right border line, between $48-73 \mathrm{~m}$ ahead, as shown in Figure (a).

\section{CONCLUSIONS AND FUTURE WORK}

In this contribution we have derived a method for estimating the free space in front of a moving vehicle, making use of radar measurements originating from stationary objects along the road side. There is no need to introduce any new sensors, since the radar sensor is already present in modern premium cars. It is just a matter of making bet- ter use of the sensor information that is already present.

Two different road border models are introduced, one linear model containing four parameters and one nonlinear model containing six parameters. These models do not depend on the fact that a radar sensor is used, implying that it is straightforward to add more sensor information from additional sensors. In other words, the approach introduced here fits well within a future sensor fusion framework, where additional sensors, such as cameras and additional radars, are incorporated.

The present approach has been evaluated on real data from both highways and rural road in Sweden. The results are encouraging and surprisingly good at times. It is of course not always perfect, but it is much more informative than just using the raw measurements. The problems typically occur when there are to too few measurements or if the measurements stems from other objects than the road side objects.

Currently there is a lot of activity within the computer vision community to be able to handle non-planar road models, making use of parametric models similar to the ones used in this paper. A very interesting avenue for future work is to combine the idea presented in this paper with information from a camera about the hight differences on the road side within a sensor fusion framework. This would probably improve the estimates, especially in situations when there are too few radar measurements available.

\section{ACKNOWLEDGEMENT}

The authors would like to thank Dr. Andreas Eidehall at Volvo Car Corporation for providing data and for initial discussions on the topic. The idea of using the arctanfunction in the predictor was brought to our attention by Professor Anders Hansson. Furthermore, we would like to thank the SEnsor Fusion for Safety (SEFS) project within the Intelligent Vehicle Safety Systems (IVSS) program and the strategic research center MOVIII, funded by the Swedish Foundation for Strategic Research (SSF) for financial support.

\section{CONTACT}

Christian Lundquist, Dr. Thomas B. Schön,

Division of Automatic Control, Department of Electrical Engineering, Linköping University, 58333 Linköping, Sweden.

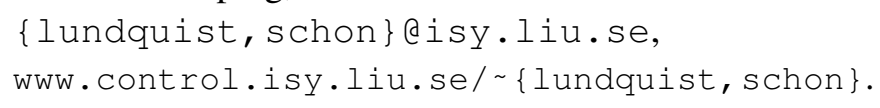




\section{REFERENCES}

1. Å Björck. Numerical methods for least squares problems. SIAM, Philadelphia, PA, USA, 1996.

2. S. Boyd and L. Vandenberghe. Convex Optimization. Cambridge University Press, 2004.

3. M. Buehler, K. Iagnemma, and S. Singh, editors. Special Issue on the 2007 DARPA Urban Challenge, Part I, volume 25 (8). Journal of Field Robotics, 2008.

4. M. Buehler, K. Iagnemma, and S. Singh, editors. Special Issue on the 2007 DARPA Urban Challenge, Part II, volume 25 (9). Journal of Field Robotics, 2008.

5. M. Buehler, K. Iagnemma, and S. Singh, editors. Special Issue on the 2007 DARPA Urban Challenge, Part III, volume 25 (10). Journal of Field Robotics, 2008.

6. E. D. Dickmanns. Dynamic Vision for Perception and Control of Motion. Springer, London, United Kingdom, 2007.

7. A. Elfes. Sonar-based real-world mapping and navigation. IEEE Journal of Robotics and Automation, 3(3):249-265, June 1987.

8. T. Fukae, N. Tamiya, and H. Mandai. Lateral distance measurement using optical spread spectrum radar. In Proceedings of the IEEE Intelligent Vehicles Symposium, pages 1-6, Tokyo, Japan, September 1996.

9. A. Gern, U. Franke, and P. Levi. Advanced lane recognition - fusing vision and radar. In Proceedings of the IEEE Intelligent Vehicles Symposimum, pages 45-51, Dearborn, MI, USA, October 2000.

10. A. Gern, U. Franke, and P. Levi. Robust vehicle tracking fusing radar and vision. In Proceedings of the international conference of multisensor fusion and integration for intelligent systems, pages 323-328, Baden-Baden, Germany, August 2001.

11. P. E. Gill, W. Murray, M. A. Saunders, and M. H. Wright. Inertia-controlling methods for general quadratic programming. SIAM Review, 33(1):136, March 1991.

12. D. Goldfarb and A. Idnani. A numerically stable dual method for solving strictly convex quadratic programs. Mathematical Programming, 27(1):1-33, 1983.

13. International Organization for Standardization ISO. Passenger cars - Test track for a severe lane-change manoeuvre - Part 1: Double lane-change. ISO 38881:1999. Geneva, Switzerland, 1999.

14. K. Kaliyaperumal, S. Lakshmanan, and K. Kluge. An algorithm for detecting roads and obstacles in radar images. IEEE Transactions on Vehicular Technology, 50(1):170-182, January 2001.
15. A. Kirchner and C. Ameling. Integrated obstacle and road tracking using a laser scanner. In Proceedings of the IEEE Intelligent Vehicles Symposium, pages 675681, Dearborn, MI, USA, October 2000.

16. A. Kirchner and T. Heinrich. Model based detection of road boundaries with a laser scanner. In Proceedings of the IEEE International Conference on Intelligent Vehicles, pages 93-98, Stuttgart, Germany, 1998.

17. S. Lakshmanan, K. Kaliyaperumal, and K. Kluge. Lexluther: an algorithm for detecting roads and obstacles in radar images. In Proceedings of the IEEE Conference on Intelligent Transportation System, pages 415-420, Boston, MA, USA, November 1997.

18. C. Lundquist and T. B. Schön. Estimation of the free space in front of a moving vehicle. Technical report, Department of Electrical Engineering, Linköping University, Sweden, September 2008.

19. C. Lundquist and T. B. Schön. Road geometry estimation and vehicle tracking using a single track model. In Proceedings of the IEEE Intelligent Vehicle Symposium, Eindhoven, The Netherlands, June 2008.

20. C. Lundquist and T. B. Schön. Road geometry estimation and vehicle tracking using a single track model. Technical Report LiTH-ISY-R-2844, Department of Electrical Engineering, Linköping University, SE58183 Linköping, Sweden, March 2008.

21. B. Ma, S. Lakshmanan, and A.O. Hero. Simultaneous detection of lane and pavement boundaries using model-based multisensor fusion. IEEE Proceedings of Intelligent Transportation Systems, 1(3):135147, September 2000.

22. R.J. Mayhan and R.A. Bishel. A two-frequency radar for vehicle automatic lateral control. IEEE Transactions on Vehicular Technology, 31(1):32-39, 1982.

23. J. C. McCall and M. M. Trivedi. Video-based lane estimation and tracking for driver assistance: Servey, system, and evaluation. IEEE Proceedings of Intelligent Transportation Systems, 7(1):20-37, March 2006.

24. M. Nikolova and A Hero. Segmentation of a road from a vehicle-mounted radar and accuracy of the estimation. In Proceedings of the IEEE Intelligent Vehicles Symposium, pages 284-289, Dearborn, MI, USA, October 2000.

25. M.J.D. Powell. On the Quadratic Programming Algorithm of Goldfrab and Idnani. Mathematical Programming Study, 25(1):46-61, 1985.

26. J. Sparbert, K. Dietmayer, and D. Streller. Lane detection and street type classification using laser range 
images. In Proceedings of the IEEE Intelligent Transportation Systems Conference, pages 454-459, Oakland, CA, USA, August 2001.

27. N. Tamiya, H. Mandai, and T. Fukae. Optical spread spectrum radar for lateral detection in vehicles. In IEEE 4th International Symposium on Spread Spectrum Techniques and Applications Proceedings, pages 195-198, Mainz, Germany, September 1996.

28. S. Thrun, W. Burgard, and D. Fox. Probabilistic Robotics. Intelligent Robotics and Autonomous Agents. The MIT Press, Cambridge, MA, USA, 2005.

29. Vägverket, Swedish Road Administration, Borlänge, Sweden. Vägar och gators utformning - Landsbygd Vägrum, 2004. 2004:80, in Swedish.

30. A. Wedel, U. Franke, H. Badino, and D. Cremers. Bspline modeling of road surfaces for freespace estimation. In Proceedings of the IEEE Intelligent Vehicles Symposium, pages 828-833, Eindhoven, The Netherlands, June 2008.

31. W. S. Wijesoma, K. R. S. Kodagoda, and A. P. Balasuriya. Road-boundary detection and tracking using ladar sensing. IEEE Transactions on Robotics and Automation, 20(3):456-464, June 2004.

32. Z. Zomotor and U. Franke. Sensor fusion for improved vision based lane recognition and object tracking with range-finders. In Proceedings of IEEE Conference on Intelligent Transportation System, pages 595-600, Boston, MA, USA, November 1997. 